\title{
Clarificação de caldo de cana-de-açúcar por peróxido de hidrogênio: efeito da presença de dextrana
}

\author{
Clarification of sugarcane juice by hydrogen peroxide: \\ effects of the presence of dextran
}

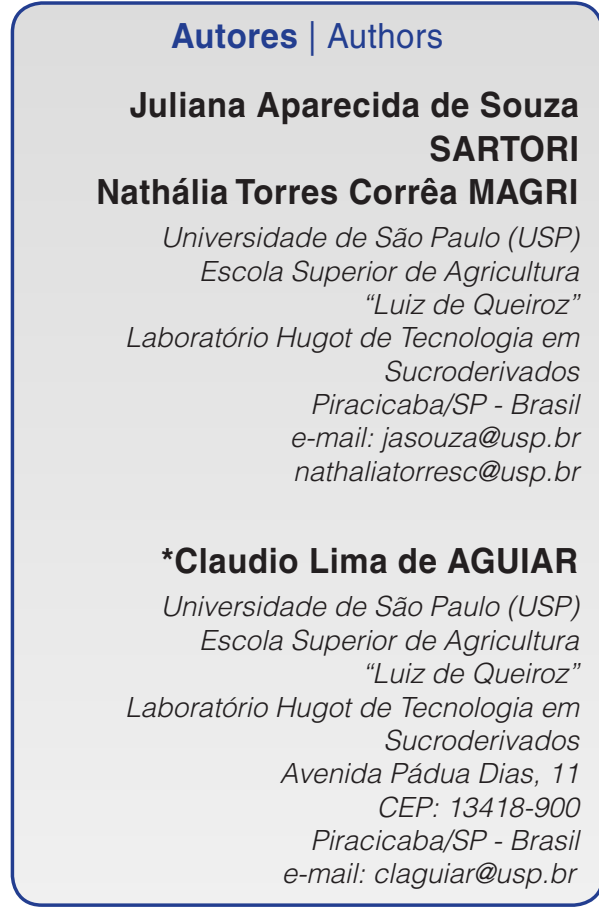

*Autor Correspondente / Corresponding Author

Recebido: Jun. 11, 2015

Aprovado: Dez. 09, 2015

\section{Resumo}

A qualidade do açúcar cristal está diretamente associada à qualidade da cana-de-açúcar (Saccharum sp.) entregue nas usinas e à eficiência do processo industrial. A dextrana é considerada um parâmetro de qualidade de matéria-prima, uma vez que sua presença indica que a cana-de-açúcar sofreu deterioração entre as etapas de corte e seu processamento. Durante o processamento do caldo, a dextrana pode interferir na eficiência do processo. Na etapa de clarificação, a sulfitação tem como principal objetivo promover a redução de cor ICUMSA do caldo de cana-de-açúcar. A cor ICUMSA é o parâmetro mais importante para a classificação comercial do açúcar no Brasil e quanto mais baixo o seu valor, mais claro é o açúcar. O peróxido de hidrogênio $\left(\mathrm{H}_{2} \mathrm{O}_{2}\right)$ tem sido estudado na clarificação do caldo de cana-de-açúcar como possível agente clarificante em substituição ao sulfito, que apresenta contra-indicações à saúde respiratória humana. Teve-se como objetivo determinar o impacto da presença de dextrana na eficiência da redução de cor ICUMSA do caldo de cana-de-açúcar por peroxidação. Durante a peroxidação, a temperatura e o $\mathrm{pH}$ influenciaram significativamente na redução da cor ICUMSA do caldo, sendo que o aumento da temperatura, aumento da dose de peróxido de hidrogênio e diminuição do pH levaram à diminuição da cor ICUMSA. Os valores de dextrana utilizados (até 1.000 ppm) não mostraram influência significativa na redução da cor do caldo de cana-de-açúcar, mas apresentaram interações significativas com os demais parâmetros.

Palavras-chave: Saccharum officinarum L.; Processos oxidativos avançados; Cor; Peroxidação; Sacarose.

\section{Summary}

The quality of crystal sugar is directly associated with the quality of the sugarcane (Saccharum s.p.) delivered to the mills and to the efficiency of the industrial process. Dextran is considered to be a quality parameter of the raw material, since its presence indicates the sugarcane suffered deterioration between the cutting and processing steps. During processing of the juice, dextran can interfere in the process efficiency. In the clarification step, the main objective of sulphiting is to promote a reduction in the ICUMSA colour of the sugarcane juice. The ICUMSA colour is the most important parameter for the classification of commercial sugar in Brazil and the lower the ICUMSA colour the whiter the sugar. Hydrogen peroxide $\left(\mathrm{H}_{2} \mathrm{O}_{2}\right)$ has been studied as a possible clarifying agent to replace sulphite in the clarification of sugarcane juice, since sulphite has some contraindications for human respiratory health. The objective of the present work was to determine the impact of the presence of dextran on the efficiency of the reduction in ICUMSA colour of sugarcane juice by peroxidation. During peroxidation, the temperature and $\mathrm{pH}$ significantly influenced the reduction in ICUMSA colour of the juice, increases in temperature, increases in the dose of hydrogen peroxide and decreases in the $\mathrm{pH}$, all leading to decreases in ICUMSA colour. The values for dextran used (up to $1,000 \mathrm{ppm}$ ) showed no significant influence on the colour of sugarcane juice, however they showed significant interactions with other parameters.

Key words: Saccharum officinarum L.; Advanced oxidation process; Colour; Peroxidation; Sucrose. 


\section{Introdução}

A dextrana representa o grupo de polissacarídeos da cana-de-açúcar formados pela ação de bactérias, principalmente Leuconostoc mesenteroides, resultantes do desdobramento dos açúcares contidos no caldo de cana-de-açúcar após a queima e/ou corte (FERNANDES, 2003). Segundo De La Rosa (1988), a presença de dextrana promove efeitos adversos na produção de açúcar branco, como alteração na polarização, aumento no consumo de cal $(\mathrm{CaO})$, deficiência na transferência de calor e aumento da viscosidade da massa cozida, que prejudica a cristalização. Na etapa de sulfitação, não há estudos na literatura sobre sua interferência. Porém essa etapa tem sido alvo de críticas por promover a formação residual de compostos reduzidos de enxofre (TRS) no açúcar. Um exemplo é descrito por Favero et al. (2011), os quais relatam que a deficiência da enzima sulfto-oxidase, que está presente naturalmente nos seres humanos, está relacionada com reações adversas sofridas pelos agentes sulfitantes. Asma ou broncoespasmos podem ser induzidos após a ingestão de alimentos com TRS, assim como indivíduos sensíveis ao sulfito podem apresentar sensação de desconforto na garganta, congestionamento no peito, tosse, hipotensão e dermatites de contato (POPOLIM, 2009; PAPAZIAN, 1996; MACHADO et al., 2008).

Novos processos têm sido propostos em substituição ao uso de enxofre na produção de açúcar cristal branco (SARTORI et al., 2014; MANE et al., 1992, 2000). Entre esses processos tem-se destacado o uso de Processos Oxidativos Avançados (POA) como o ozônio e o peróxido de hidrogênio $\left(\mathrm{H}_{2} \mathrm{O}_{2}\right)$, que já são empregados em diferentes setores da indústria de alimentos. O uso do $\mathrm{H}_{2} \mathrm{O}_{2}$ é considerado de baixo custo e não exige grandes modificações na infraestrutura industrial, o que o torna ainda mais atrativo na indústria do açúcar (MADSEN et al., 1978). O peróxido de hidrogênio é certificado pela Food and Drug Administration (FDA) como um produto GRAS (Generally Recognized As Safe) quando utilizado como agente de branqueamento em alimentos desde 1979 (FDA, 2015). No tratamento do caldo de cana-de-açúcar com $\mathrm{H}_{2} \mathrm{O}_{2}$, há reduções significativas nos teores de aminoácidos, açúcares redutores, amido e polifenóis, ocorrendo ainda diminuição nos teores de cinzas e da viscosidade, bem como aumento na pureza do xarope pela remoção de não-açúcares (MADSEN et al., 1978; MANE et al., 1998, 2000). Segundo Mane et al. (2000), com o uso de $\mathrm{H}_{2} \mathrm{O}_{2}$ há predominância de reações irreversíveis de decomposição de compostos pigmentosos e a cor do açúcar tratado permanece sem sofrer alterações por longos períodos de armazenamento.

Estudos anteriores determinaram os efeitos do peróxido de hidrogênio na composição do caldo de cana-de-açúcar. O peróxido de hidrogênio promoveu a sedimentação de não açúcares do caldo de cana-deaçúcar, sem degradação da sacarose (SARTORI et al., 2015). Com o uso de um planejamento fatorial é possível avaliar as influências de diferentes variáveis experimentais de interesse e os efeitos de interação na resposta (TEÓFILO et al., 2006). Portanto, esse trabalho teve como objetivo avaliar a influência da presença de dextrana no caldo de cana-de-açúcar, sob diferentes condições reacionais de temperatura, $\mathrm{pH}$ e doses de $\mathrm{H}_{2} \mathrm{O}_{2}$ sobre a redução de cor ICUMSA em caldo de cana-de-açúcar tratado por peroxidação.

\section{Material e métodos}

\subsection{Colheita da cana-de-açúcar e preparação das amostras de caldo}

A cana-de-açúcar utilizada nesta etapa foi a variedade SP 81-3250, proveniente de plantação no município de Piracicaba, SP (2242'S, 47³8'W e 596 m de altitude), na Estação Experimental da Escola Superior de Agricultura Luiz de Queiroz, Universidade de São Paulo (ESALQ-USP). O caldo foi obtido com o auxílio de uma moenda Modelo Mausa (Piracicaba, SP), sendo peneirado e acondicionado em frascos de polipropileno opaco, em freezer $\mathrm{a}-18 \pm 2^{\circ} \mathrm{C}$, em frações homogêneas e subdivididas para os ensaios experimentais e amostragem analítica.

\subsection{Ensaios de clarificação do caldo de cana-de-açúcar}

Ensaios de clarificação do caldo de cana-deaçúcar utilizando $\mathrm{H}_{2} \mathrm{O}_{2}$ foram estruturados através de planejamento fatorial completo $2^{4}$ rotacionado, com 3 pontos centrais e 8 pontos axiais, com o intuito de obter as melhores condições para redução de cor ICUMSA do caldo de cana-de-açúcar pela solução de peróxido de hidrogênio a $35 \%(\mathrm{~m} / \mathrm{m})$. Os fatores estudados foram $\mathrm{pH}$, temperatura $\left({ }^{\circ} \mathrm{C}\right), \mathrm{H}_{2} \mathrm{O}_{2}$ (ppm) e dextrana (ppm). Cada ensaio foi desenvolvido em reator de vidro com capacidade para $600 \mathrm{~mL}$, sendo utilizados $400 \mathrm{~mL}$ de caldo de cana-de-açúcar. Os reatores foram colocadas em shaker sob rotações constantes (100 rpm) durante uma hora. Os valores utilizados nos ensaios realizados estão descritos na Tabela 1.

A adição de solução de catalase de fígado bovino (2.300 UI) foi feita para paralisação da atividade reacional do $\mathrm{H}_{2} \mathrm{O}_{2}$. Amorim et al. (2002) afirmam que a enzima catalisa a decomposição do peróxido de hidrogênio em água e oxigênio, acarretando na paralisação da sua ação reativa. Ao final, as amostras foram devidamente armazenadas em recipientes de polipropileno opaco e, posteriormente, submetidas às análises laboratoriais. 
Clarificação de caldo de cana-de-açúcar por peróxido de hidrogênio: efeito da presença de dextrana

SARTORI, J. A. S. et al.

Tabela 1. Valores codificados e reais dos parâmetros do planejamento fatorial completo 24 rotacionado, com 3 pontos centrais e 8 pontos axiais.

\begin{tabular}{lccccc}
\multicolumn{1}{c}{ Parâmetros } & $-\alpha$ & $\mathbf{- 1}$ & $\mathbf{0}$ & $\mathbf{+ 1}$ & $\mathbf{+}$ \\
$\mathrm{pH}$ & 1,0 & 3,0 & 5,0 & 7,0 & 9,0 \\
Temperatura $\left({ }^{\circ} \mathrm{C}\right)$ & 10 & 30 & 50 & 70 & 90 \\
$\mathrm{H}_{2} \mathrm{O}_{2}$ (ppm) & 0 & 300 & 600 & 900 & 1200 \\
Dextrana & 0 & 250 & 500 & 750 & 1000 \\
\hline
\end{tabular}

\subsection{Análise de cor ICUMSA}

Para medir o índice de cor ICUMSA (Method G.S. 2/3-9) (ICUMSA, 2011), fez-se uma diluição preliminar do caldo de cana-de-açúcar até 1,25 Brix de sólidos solúveis totais (SST). As amostras foram filtradas a vácuo através de membrana de PTFE com 0,45 $\mu \mathrm{m}$ de diâmetro de poro (Millipore Co., São Paulo, Brasil) e o $\mathrm{pH}$, ajustado para 7,0 com $\mathrm{HCl}$ (grau analítico) ou $\mathrm{NaOH}$ 0,05M. Em seguida, a absorbância foi medida a 420 nm em espectrofotômetro UV mini 1240 (Shimadzu Co., Kyoto, Japão). O índice de cor ICUMSA foi expresso a partir da Equação 1, na qual $\rho$ : densidade da amostra (determinada pela Equação 2; Brix: concentração de SST na amostra original; Brix : concentração de SST na amostra diluída); Abs $_{420 \mathrm{~nm}}$ (absorbância a 420 nm).

Cor ICUMSA $=\left[\left(\right.\right.$ Abs $\left._{420 \mathrm{~nm}} \times 1000\right) /\left(\rho \times\left(\right.\right.$ Brix $\left.\left.\left._{c} / 100\right)\right)\right]$

$\rho=1+\left[\left(\left(\right.\right.\right.$ Brix $_{o} \times\left(200+\right.$ Brix $\left.\left.\left._{0}\right)\right) / 54000\right) \times\left(\right.$ Brix $_{d} /$ Brix $\left.\left._{o}\right)\right]$

\subsection{Análise estatística dos dados}

Foi realizado tratamento dos resultados da variável dependente (cor ICUMSA) por análise de regressão múltipla da metodologia de superfície de resposta. Para testar a adequação do modelo obtido foi realizada análise da variância (ANOVA). O teste $\mathrm{F}$ e o coeficiente de determinação foram utilizados para determinar a significância do modelo, de acordo com Barros Neto et al. (1995). Quando a ANOVA mostrou-se significativa, gráficos de superfície de respostas foram construídos para visualizar os efeitos das variáveis independentes e de suas interações sobre a resposta avaliada, sendo o processamento dos dados realizado com o programa Statistica (versão 12, Statsoft).

\section{Resultados e discussão}

Na Tabela 2 são apresentados os resultados de cor ICUMSA do caldo de cana-de-açúcar tratados com peróxido de hidrogênio, seguindo a planilha de planejamento fatorial completo $2^{4}$, com 16 pontos fatoriais, 3 centrais e 8 axiais.

Os coeficientes de regressão a 5\% de significância dos fatores lineares $(L)$ e quadráticos $(Q)$ e das interações são apresentados na Tabela 3. Os fatores pH (L), pH (Q), temperatura $(\mathrm{L})$, temperatura $(\mathrm{Q}), \mathrm{H}_{2} \mathrm{O}_{2}(\mathrm{~L}), \mathrm{H}_{2} \mathrm{O}_{2}(\mathrm{Q})$, dextrana (Q) e as interações $\mathrm{pH} \times \mathrm{H}_{2} \mathrm{O}_{2}$, temperatura $\times$ $\mathrm{H}_{2} \mathrm{O}_{2}$, temperatura $\times$ dextrana, $\mathrm{H}_{2} \mathrm{O}_{2} \times$ dextrana foram significativos ao intervalo de confiança de 95\%.

A variável $\mathrm{pH}(\mathrm{L})$ e as interações temperatura $\times$ $\mathrm{H}_{2} \mathrm{O}_{2}$ e $\mathrm{H}_{2} \mathrm{O}_{2} \times$ dextrana apresentaram efeitos positivos na resposta da cor ICUMSA do caldo, indicando que um aumento em qualquer um dos fatores contribui para a elevação na cor ICUMSA no caldo. Temperatura ( $\mathrm{L}), \mathrm{H}_{2} \mathrm{O}_{2}$ (L) e interação $\mathrm{pH} \times \mathrm{H}_{2} \mathrm{O}_{2}$ apresentam efeitos negativos na resposta da cor ICUMSA, ou seja, com o aumento dos fatores ocorre diminuição na cor.

Os parâmetros $\mathrm{pH}$, temperatura, $\mathrm{H}_{2} \mathrm{O}_{2}$ e dextrana (Q) apresentaram efeitos com valores positivos, indicando a existência de uma região de mínima e a superfície de resposta, na forma de uma parábola positiva, isto é, com a curvatura voltada para cima.

Observa-se que, dos 14 parâmetros do modelo nos efeitos estimados dos coeficientes de regressão dos fatores lineares, quadráticos e interações, 10 foram significativos $(p \leq 0,05)$ e permaneceram no modelo para a construção da análise de variância (ANOVA) ajustada (Tabela 4).

O modelo de regressão gerado foi significativo $(p \leq 0,05)$, uma vez que o $F_{\text {calculado }(0,95 ; 11 ; 15)}=2,956$ foi maior que $\circ F_{\text {tabelado }(0,95 ; 11 ; 15)}=2,51$. A falta de ajuste também foi significativa, uma vez que o $F_{\text {calculado }(0,95 ; 13 ; 2)}=78,65$ foi maior que o $F_{\text {tabelado }(0,95 ; 13 ; 2)}=78,65$. Segundo Barros Neto et al. (1995), o valor alto para a falta de ajuste pode ser justificado pelo fato da média dos pontos centrais ser muito próxima e o erro puro muito baixo, o que corrobora os resultados obtidos (Tabela 4).

O coeficiente de determinação da regressão $\left(R^{2}\right)$ mede a proporção da variação total da resposta que é explicada pelo modelo. Desse modo, quanto maior o $\mathrm{R}^{2}$, ou seja mais próximo de 1, menor será o erro e melhor será o modelo. Quando o valor do $\mathrm{R}^{2}$ for menor que 0,60, os modelos só poderão ser utilizados como indicadores de tendências, não podendo ser utilizados para fins preditivos (Barros Neto et al., 1995). O coeficiente de determinação da regressão $\left(R^{2}\right)$ do modelo obtido foi igual à 0,684 , ou seja, $68,4 \%$ da variância total em torno da média é explicada pelo modelo. 
Clarificação de caldo de cana-de-açúcar por peróxido de hidrogênio: efeito da presença de dextrana

SARTORI, J. A. S. et al.

Tabela 2. Resultados de cor ICUMSA obtidos experimentalmente através da peroxidação do caldo de cana-de-açúcar.

\begin{tabular}{|c|c|c|c|c|c|}
\hline \multirow{2}{*}{$\begin{array}{l}\mathrm{N}^{\circ} \text { ensaios } \\
\text { (Tratamento) }\end{array}$} & \multicolumn{4}{|c|}{ Níveis de variáveis independentes codificados e reais } & \multirow{2}{*}{$\begin{array}{c}\text { Resposta } \\
\text { cor ICUMSA (UI) }\end{array}$} \\
\hline & $\mathrm{X}_{1} \mathrm{pH}$ & $\mathrm{X}_{2}$ temperatura $\left({ }^{\circ} \mathrm{C}\right)$ & $\mathrm{X}_{3} \mathrm{H}_{2} \mathrm{O}_{2}(\mathrm{ppm})$ & $\mathrm{X}_{4}$ dextrana (ppm) & \\
\hline 1 & $-1(3,0)$ & $-1(30)$ & $-1(300)$ & $-1(250)$ & $3732,4 \pm 154,8$ \\
\hline 2 & $+1(7,0)$ & $-1(30)$ & $-1(300)$ & $-1(250)$ & $3622,8 \pm 71,0$ \\
\hline 3 & $-1(3,0)$ & $+1(70)$ & $-1(300)$ & $-1(250)$ & $2628,3 \pm 122,6$ \\
\hline 4 & $+1(7,0)$ & $+1(70)$ & $-1(300)$ & $-1(250)$ & $4329,8 \pm 136,6$ \\
\hline 5 & $-1(3,0)$ & $-1(30)$ & $+1(900)$ & $-1(250)$ & $2664,0 \pm 149,7$ \\
\hline 6 & $+1(7,0)$ & $-1(30)$ & $+1(900)$ & $-1(250)$ & $3092,7 \pm 152,1$ \\
\hline 7 & $-1(3,0)$ & $+1(70)$ & $+1(900)$ & $-1(250)$ & $2962,6 \pm 104,1$ \\
\hline 8 & $+1(7,0)$ & $+1(70)$ & $+1(900)$ & $-1(250)$ & $3386,0 \pm 108,7$ \\
\hline 9 & $-1(3,0)$ & $-1(30)$ & $-1(300)$ & $+1(750)$ & $3081,1 \pm 140,6$ \\
\hline 10 & $+1(7,0)$ & $-1(30)$ & $-1(300)$ & $+1(750)$ & $4029,5 \pm 42,7$ \\
\hline 11 & $-1(3,0)$ & $+1(70)$ & $-1(300)$ & $+1(750)$ & $2865,0 \pm 163,6$ \\
\hline 12 & $+1(7,0)$ & $+1(70)$ & $-1(300)$ & $+1(750)$ & $3717,5 \pm 40,5$ \\
\hline 13 & $-1(3,0)$ & $-1(30)$ & $+1(900)$ & $+1(750)$ & $3448,7 \pm 157,4$ \\
\hline 14 & $+1(7,0)$ & $-1(30)$ & $+1(900)$ & $+1(750)$ & $3922,3 \pm 299,4$ \\
\hline 15 & $-1(3,0)$ & $+1(70)$ & $+1(900)$ & $+1(750)$ & $3511,8 \pm 187,4$ \\
\hline 16 & $+1(7,0)$ & $+1(70)$ & $+1(900)$ & $+1(750)$ & $3841,7 \pm 111,7$ \\
\hline 17 & $-\alpha(1,0)$ & $0(50)$ & $0(600)$ & $0(500)$ & $3664,2 \pm 106,9$ \\
\hline 18 & $+\alpha(9,0)$ & $0(50)$ & $0(600)$ & $0(500)$ & $4072,1 \pm 163,9$ \\
\hline 19 & $0(5,0)$ & $-\alpha(10)$ & $0(600)$ & $0(500)$ & $3356,0 \pm 125,8$ \\
\hline 20 & $0(5,0)$ & $+\alpha(90)$ & $0(600)$ & $0(500)$ & $2770,3 \pm 70,3$ \\
\hline 21 & $0(5,0)$ & $0(50)$ & $-\alpha(0)$ & $0(500)$ & $3213,5 \pm 144,2$ \\
\hline 22 & $0(5,0)$ & $0(50)$ & $+\alpha(1200)$ & $0(500)$ & $3098,3 \pm 37,4$ \\
\hline 23 & $0(5,0)$ & $0(50)$ & $0(600)$ & $-\alpha(0)$ & $4296,9 \pm 50,2$ \\
\hline 24 & $0(5,0)$ & $0(50)$ & $0(600)$ & $+\alpha(1000)$ & $3018,5 \pm 50,2$ \\
\hline 25 & $0(5,0)$ & $0(50)$ & $0(600)$ & $0(500)$ & $2912,4 \pm 187,1$ \\
\hline 26 & $0(5,0)$ & $0(50)$ & $0(600)$ & $0(500)$ & $2865,1 \pm 107,9$ \\
\hline 27 & $0(5,0)$ & $0(50)$ & $0(600)$ & $0(500)$ & $2823,7 \pm 99,5$ \\
\hline
\end{tabular}

Condições reacionais estabelecidas: tempo $=1$ h; agitação $=100$ rpm; Brix do caldo: $20,7^{\circ}$.

Tabela 3. Estimativa do coeficiente de regressão e teste T sobre a resposta de cor ICUMSA.

\begin{tabular}{|c|c|c|c|}
\hline \multirow{2}{*}{ Variáveis independentes } & \multicolumn{3}{|c|}{ Coeficientes estimados } \\
\hline & Coeficiente & Teste T & Erro puro \\
\hline Média & $2867,07^{\star}$ & $111,89^{*}$ & $0,00^{*}$ \\
\hline $\mathrm{pH}(\mathrm{L})$ & $470,15^{\star}$ & $25,95^{\star}$ & $0,00^{*}$ \\
\hline $\mathrm{pH}(\mathrm{Q})$ & $492,92^{*}$ & $25,65^{\star}$ & $0,00^{*}$ \\
\hline Temperatura (L) & $-108,32^{*}$ & $-5,98^{*}$ & $0,03^{*}$ \\
\hline Temperatura (Q) & $90,42^{*}$ & $4,70^{*}$ & $0,04^{*}$ \\
\hline $\mathrm{H}_{2} \mathrm{O}_{2}(\mathrm{~L})$ & $-98,72^{\star}$ & $-5,45^{\star}$ & $0,03^{*}$ \\
\hline $\mathrm{H}_{2} \mathrm{O}_{2}(\mathrm{Q})$ & $136,80^{*}$ & $7,12^{*}$ & $0,02^{*}$ \\
\hline Dextrana $(\mathrm{L})$ & $-65,02$ & $-3,59$ & 0,07 \\
\hline Dextrana $(Q)$ & $387,70^{*}$ & $20,17^{*}$ & $0,00^{*}$ \\
\hline $1 \mathrm{~L} \times 2 \mathrm{~L}$ & $223,58^{*}$ & $10,07^{*}$ & $0,01^{*}$ \\
\hline $1 \mathrm{~L} \times 3 \mathrm{~L}$ & $-189,35^{\star}$ & $-8,53^{*}$ & $0,01^{*}$ \\
\hline $1 \mathrm{~L} \times 4 \mathrm{~L}$ & $-7,75$ & $-0,35$ & 0,76 \\
\hline $2 \mathrm{~L} \times 3 \mathrm{~L}$ & $159,65^{\star}$ & $7,19^{*}$ & $0,02^{*}$ \\
\hline $2 \mathrm{~L} \times 4 \mathrm{~L}$ & $-64,75$ & $-2,92$ & 0,10 \\
\hline $3 \mathrm{~L} \times 4 \mathrm{~L}$ & $432,73^{\star}$ & $19,50^{*}$ & $0,00^{*}$ \\
\hline
\end{tabular}

\footnotetext{
${ }^{*}$ Significativo a $p \leq 0,05$.
} 
Tabela 4. Análise da variância (ANOVA) do modelo ajustado $(p \leq 0,05)$ obtida a partir do coeficiente de regressão da resposta de cor ICUMSA.

\begin{tabular}{lcccc}
\multicolumn{1}{c}{ FV } & SQ & GL & MQ & Teste F \\
Regressão & 4374375 & 11 & 397670,5 & $2,956^{*}$ \\
Resíduo & 2017997 & 15 & 134533,1 & $78,65^{*}$ \\
Falta de ajuste & 2014058 & 13 & 154927,5 & \\
Erro puro & 3940 & 2 & 1969,8 & \\
Total & 6392373 & 26 & & \\
\hline
\end{tabular}

*Significativo a $p \leq 0,05$.

Os coeficientes de regressão significativos a $95 \%$ foram considerados nos modelos matemáticos propostos para representar a equação de cor ICUMSA em função do $\mathrm{pH}$, temperatura, $\mathrm{H}_{2} \mathrm{O}_{2}$ e dextrana. Segue abaixo a Equação 3, na qual $\mathrm{A}=\mathrm{pH} ; \mathrm{B}=$ temperatura; $\mathrm{C}=\mathrm{H}_{2} \mathrm{O}_{2}$; $\mathrm{D}=$ dextrana.

Cor $=2867,07+235,08^{*} A+246,5^{*} A^{2}-54,16^{*} B+$ $45,21 * B^{2}-49,36 * C+68,40 * C^{2}+193,85^{*} D^{2}+$

$111,79^{*} A^{*} B-94,68^{*} A^{*} C+79,83^{*} B^{*} C+216,36^{*} C^{*} D$

Através das análises de superfícies de resposta puderam-se visualizar os efeitos das variáveis independentes e de suas interações sobre a resposta avaliada (cor ICUMSA), uma vez que a ANOVA mostrou-se significativa.

Observando-se a superfície de resposta (Figura 1a), verificou-se que os menores valores de cor ICUMSA foram obtidos em condições de elevada quantidade de peróxido de hidrogênio (maior que 600 ppm) combinadas com valores de dextrana entre 250 e 750 ppm.

Os menores valores de cor ICUMSA foram obtidos em $\mathrm{pH}$ abaixo de 7 , independentemente da quantidade de peróxido de hidrogênio utilizada (0 a 1.200 ppm). Porém, para valores de $\mathrm{pH}$ acima de 7 , somente com o aumento da quantidade de peróxido houve tendência da diminuição da cor final (Figura 1b).

Considerando a superfície de resposta para interação das variáveis $\mathrm{pH}$ e peróxido de hidrogênio (Figura 2a), em temperatura menores que $50{ }^{\circ} \mathrm{C}$ foi necessária uma maior quantidade de peróxido de hidrogênio (maior que 300 ppm) para obter baixos valores de cor ICUMSA. Em temperaturas maiores que $50{ }^{\circ} \mathrm{C}$, menores quantidades de peróxido de hidrogênio (menor que 300 ppm) já foram suficiente para obter menores índices de cor ICUMSA.

Os menores valores de cor ICUMSA foram obtidos em pH menores que 7, ou seja, ácidos. Com relação à temperatura, a cor ICUMSA final foi menor na faixa de temperatura de 30 a $70{ }^{\circ} \mathrm{C}$ quando em $\mathrm{pH}$ abaixo de 7 (Figura 2b). Em pH acima de 7, os valores de cor ICUMSA finais foram maiores independentemente da temperatura $\left(10\right.$ a $\left.90{ }^{\circ} \mathrm{C}\right)$.
Pela análise da interação de pH × dextrana foi possível observar que os menores valores de cor ICUMSA foram obtidos em valores de $\mathrm{pH}$ menor que $7 \mathrm{e}$ quantidades de dextrana entre 250 e 750 ppm. Em pH acima de 7 , elevados valores de cor final foram obtidos independentemente do valor de dextrana utilizado (Figura 3a). Na Figura 3b é possível observar que os menores valores de cor ICUMSA finais foram obtidos em valores de dextrana entre 250 e 750 ppm.

De acordo com estudos realizados por Clarke (1997), quantidades de dextrana em torno de 300 ppm foram suficientes para promover problemas na polarização (teor aparente de sacarose) do açúcar bruto. A polarização do açúcar define a porcentagem de sacarose no açúcar e é expressa em ${ }^{\circ} Z$ (graus Zucker), sendo esse valor sempre superior a 99,7\% para açúcar de consumo direto (OLIVEIRA et al., 2007). Alves et al. (2013, 2014) também mostraram mudanças nos valores da polarização devido à adição de diferentes doses de dextrana. A presença de dextrana superestimou os valores de sacarose presentes no caldo de cana-de-açúcar, sendo que valores maiores que 100 ppm foram suficientes para promover essas alterações. Além do efeito na polarização, a dextrana causa aumento na viscosidade do caldo de cana-de-açúcar e resulta em caldo de cana-de-açúcar turvo, devido à dificuldade de separar os materiais em suspensão (DE LA ROSA, 1988). No entanto, neste estudo, a quantidade de dextrana utilizada não apresentou efeito significativo na resposta final (cor ICUMSA).

A menor cor ICUMSA foi obtida quando o $\mathrm{pH}$ do caldo de cana-de-açúcar foi menor que 7, como indicado pela análise dos coeficientes de regressão, o que é confirmado em trabalho apresentado por Rathi et al. (2003), no qual afirma-se que no uso do peróxido de hidrogênio, em $\mathrm{pH}$ ácidos, o OH·é o oxidante reativo predominante, porém, em $\mathrm{pH}$ alcalino, radicais hidroperóxidos são formados pela reação dos radicais hidroxílicos com o peróxido de hidrogênio, sendo que esses radicais hidroperóxidos não têm alto poder de oxidação, como o OH-. Nguyen e Dohert (2012) avaliaram a degradação do ácido cafeico utilizando o peróxido de hidrogênio. O ácido cafeico foi escolhido para representar os compostos fenólicos e precursores de cor presentes 
Clarificação de caldo de cana-de-açúcar por peróxido de hidrogênio: efeito da presença de dextrana SARTORI, J. A. S. et al.
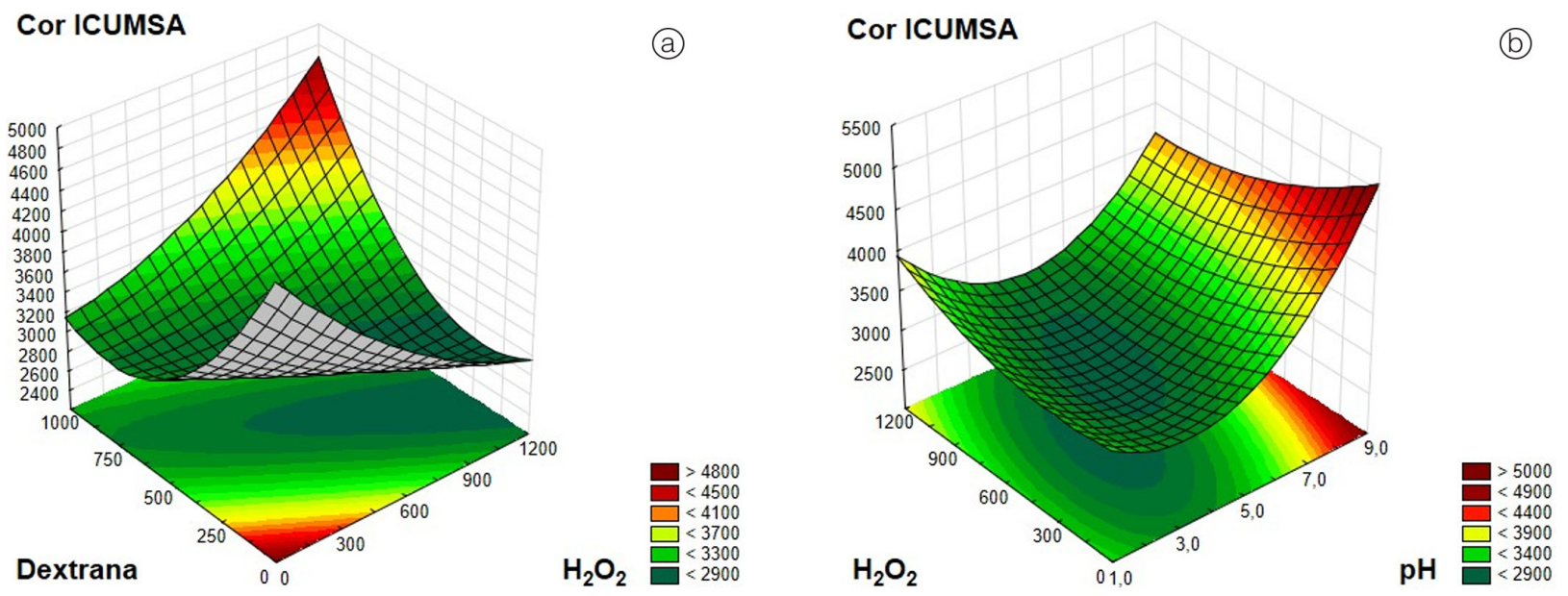

Figura 1. (a) Superfície de resposta para interação das variáveis X3: peróxido de hidrogênio $\left(\mathrm{H}_{2} \mathrm{O}_{2}\right)$ e X4: dextrana para obtenção de menores valores de cor ICUMSA; (b) Superfície de resposta para interação das variáveis X1: pH e X3: peróxido de hidrogênio $\left(\mathrm{H}_{2} \mathrm{O}_{2}\right)$ para obtenção de menores valores de cor ICUMSA através da peroxidação.
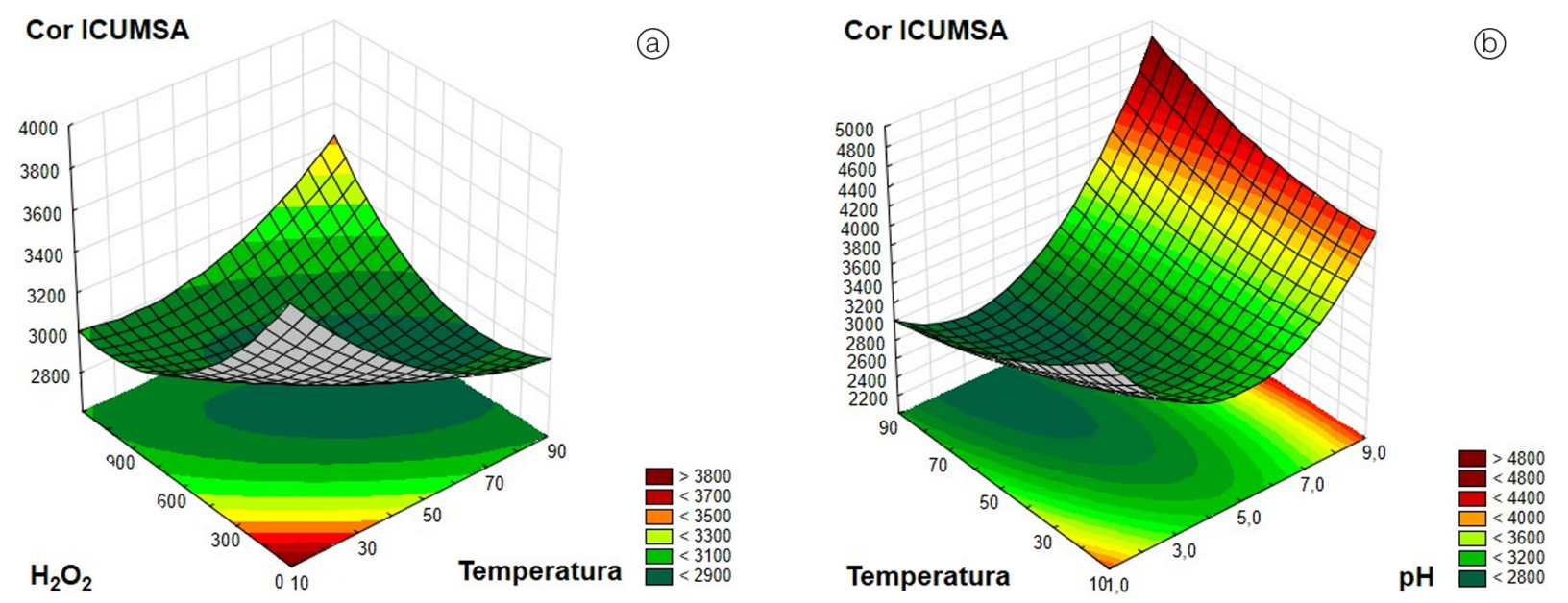

Figura 2. (a) Superfície de resposta para interação das variáveis $X 3$ : peróxido de hidrogênio $\left(\mathrm{H}_{2} \mathrm{O}_{2}\right)$ e $\mathrm{X} 2$ : temperatura para obtenção de menores valores de cor ICUMSA; (b) Superfície de resposta para a interação das variáveis X1: pH e X2: temperatura para obtenção de menores valores de cor ICUMSA através da peroxidação.

\section{Cor ICUMSA}

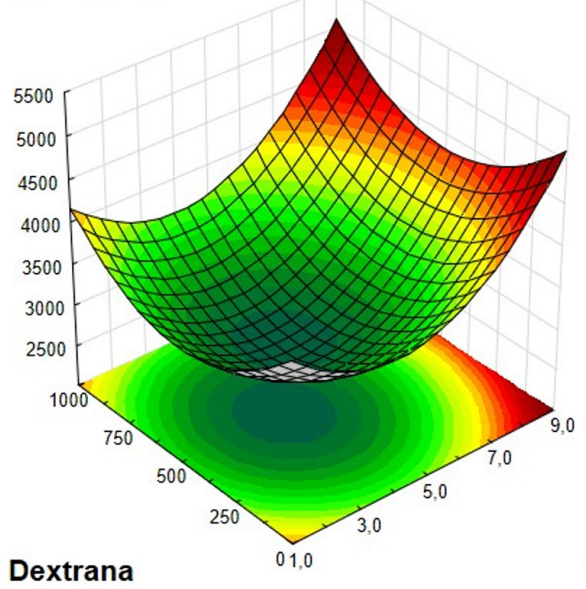

(a)

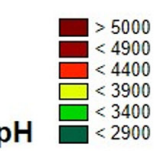

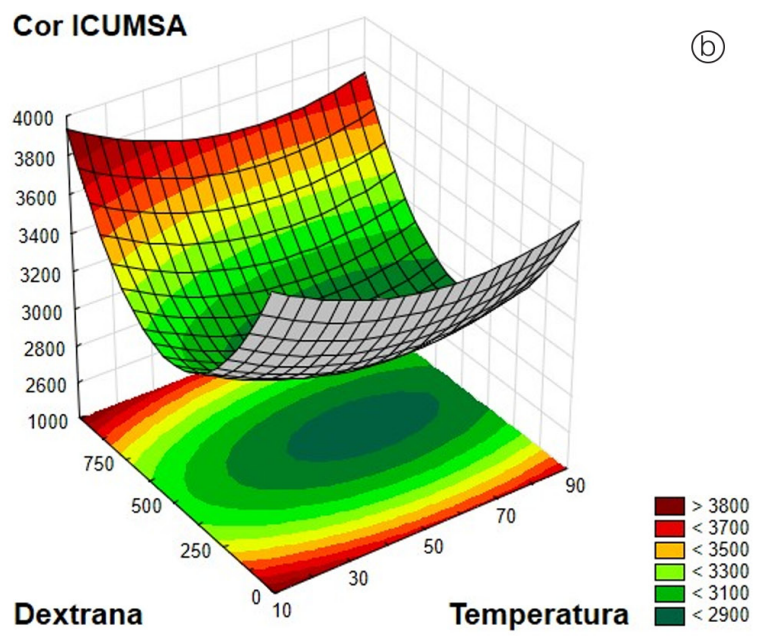

Figura 3. (a) Superfície de resposta para a interação das variáveis X4: dextrana e X1: pH para obtenção de menores valores de cor ICUMSA para obtenção de menores valores de cor ICUMSA; (b) Superfície de resposta para interação das variáveis X4: dextrana e X2: temperatura para obtenção de menores valores de cor ICUMSA através da peroxidação. 
Clarificação de caldo de cana-de-açúcar por peróxido de hidrogênio: efeito da presença de dextrana

SARTORI, J. A. S. et al.

no caldo de cana-de-açúcar. Em pH igual a 3 e com 60 minutos de reação, em temperatura ambiente, houve degradação de $16,5 \%$ do ácido cafeico utilizando-se 100 ppm de $\mathrm{H}_{2} \mathrm{O}_{2}$. Nos valores de pH 4 e 5 não foi observada degradação do ácido cafeico. De maneira similar, os estudos de Mandro et al. (2015) demonstraram que o peróxido de hidrogênio foi efetivo na degradação de rutina (utilizada para simular as impurezas pigmentosas presentes no caldo de cana-de açúcar), sendo que sua degradação foi maior com o aumento da quantidade de peróxido de hidrogênio utilizada.

Observando-se as figuras e os coeficientes de regressão é possível afirmar que o aumento da temperatura (até $70^{\circ} \mathrm{C}$ ) promove uma maior redução da cor ICUMSA do caldo tratado com peróxido de hidrogênio. Mane et al. (1992) obtiveram em seu trabalho a redução de $6,5 \%$ do valor da cor ICUMSA do caldo tratado com peróxido de hidrogênio $(0,1 \%, \mathrm{~m} / \mathrm{m})$ em comparação com o caldo que não foi tratado, sendo que ambos foram aquecidos até $70{ }^{\circ} \mathrm{C}$ e caleados, com a adição de leite de cal a $12^{\circ}$ Brix. Mane et al. (1992) também observaram redução de aminoácidos, polifenóis e açúcares redutores no caldo peroxidado.

\section{Conclusão}

A redução da cor ICUMSA do caldo de cana-deaçúcar tratado com peróxido de hidrogênio foi maior em temperaturas mais elevadas (maiores que $50^{\circ} \mathrm{C}$ ), com maiores doses de $\mathrm{H}_{2} \mathrm{O}_{2}$ (>600 ppm) e em valores de pH menores que 7, ou seja, em meio ácido. A dose de dextrana utilizada não foi significativa estatisticamente na redução da cor do caldo, porém apresentou interação significativa com os demais parâmetros.

\section{Agradecimentos}

Os autores agradecem à Fundação Amparo à Pesquisa do Estado de São Paulo (FAPESP 2009/54635-1; 2012/15618-7) e ao Conselho Nacional de Desenvolvimento Científico e Tecnológico (CNPq 506328/2010-4) pelo apoio financeiro.

\section{Referências}

ALVES, F. V.; AGUIAR, C. L.; SARMENTO, S. B. S. The effects of starch and dextran on technological analyses of sugarcane juice. Sugar Journal, New Orleans, v. 76, n. 3, p. 14-23, 2013.

ALVES, F. V.; POLESI, L. F.; AGUIAR, C. L.; SARMENTO, S. B. Structural and physicochemical characteristics of starch from sugar cane and sweet sorghum stalks. Carbohydrate Polymers, Barking, v. 111, p. 592-597, 2014. http://dx.doi.org/10.1016/j. carbpol.2014.05.034. PMid:25037392.

AMORIM, A. M.; GASQUES, M. D. G.; ANDREUS, J.; SCHARF, $M$. The application of catalase for the elimination of hydrogen peroxide residues after bleaching of cotton fabrics. Anais da Academia Brasileira de Ciências, Rio de Janeiro, v. 74, n. 3, p. 433-436, 2002.

BARROS NETO, B.; SCARMINIO, I. S.; BRUNS, R. E. Planejamento e otimização de experimentos. Campinas: Editora da Unicamp, 1995. 299 p.

CLARKE, A. M. Dextrans en los ingenios azucares: presencia y control. Sugar y Azucar, New York, v. 92, p. 38-45, 1997.

DE LA ROSA, R. D. Las dextrans: su efecto sobre la polarización de la sacarosa y la economia azucarera. International Sugar Journal, London, v. 100, n. 1191S, p. 103-145, 1988.

FAVERO, D. M.; RIBEIRO, C. S. G.; AQUINO, A. D. Sulfitos: importância na indústria alimentícia e seus possíveis malefícios à população. Segurança Alimentar e Nutricional, Campinas, v. 18, n. 1, p. 1-20, 2011.

FERNANDES, A. C. Cálculos na agroindústria da cana-deaçúcar. 2. ed. Piracicaba: EME, 2003. 240 p.

FOOD AND DRUG ADMINISTRATION - FDA. Select Committee on GRAS Substances (SCOGS) opinion: hydrogen peroxide. Silver Spring: FDA, 2015. Disponível em: <http://www.fda. gov/Food/IngredientsPackagingLabeling/GRAS/SCOGS/ ucm260427.htm>. Acesso em: 11 nov. 2015.

INTERNATIONAL COMISSION FOR UNIFORM METHODS OF SUGAR ANALYSIS - ICUMSA. Method G.S. 2/3-9: the determination of sugar solution colour at 7.0. Lincolnshire: ICUMSA, 2011.

MACHADO, R. M. D.; TOLEDO, M. C. F.; ALMEIDA, C. A. S.; VICENTE, E. Analytical determination of sulphites in fruit juices available on the Brazilian market. Brazilian Journal of Food Technology, Campinas, v. 11, n. 3, p. 226-233, 2008.

MADSEN, R. F.; KOTFOD-NIELSEN, W.; WINSTROM-OLSEN, B.; NIELSEN, T. E. Formation of color compounds in production of sugar from sugar beet. Sugar Technology Reviews, Amsterdam, v. 6, n. 1, p. 49-115, 1978.

MANDRO, J. L.; BRAGA, N. L. L.; CATELAN, M. G.; BAPTISTA, A. S.; SARTORI, J. A. S.; CORREA, N. T.; ROCHA, A. L. B.; LIMA, R. B.; AGUIAR, C. L. Degradação de rutina em sistemas-modelo de caldo de cana-de-açúcar pela ação de peróxido de hidrogênio. Revista de Quimica Industrial, Rio de Janeiro, 2015. No prelo.

MANE, J. D.; PACHPUTE, S. P.; PHADNIS, S. P. Effects of hydrogen peroxide treatment on cane syrup. International Sugar Journal, London, v. 1193, n. 100, p. 210-212, 1998.

MANE, J. D.; PHADNIS, S. P.; JADHAV, S. J. Effects of hydrogen peroxide on cane juice constituents. International Sugar Journal, London, v. 94, n. 1128, p. 322-324, 1992.

MANE, J. D.; PHADNIS, S. P.; JAMBHALE, D. B.; YEWALE, A. $V$. Mill scale evaluation of hydrogen peroxide as a processing aid: quality improvement in plantation white sugar. International Sugar Journal, London, v. 1222, n. 102, p. 530-533, 2000. 
Clarificação de caldo de cana-de-açúcar por peróxido de hidrogênio: efeito da presença de dextrana

SARTORI, J. A. S. et al.

NGUYEN, D. M. T.; DOHERT, W. O. S. Phenolics in sugar cane juice: potential degradation by hydrogen peroxide and Fenton's reagent. International Sugar Journal, London, v. 114, p. 309-315, 2012.

OLIVEIRA, D. T.; ESQUIAVETO, M. M. M.; SILVA JÚNIOR, J. F. Impacto dos itens da especificação do açúcar na indústria alimentícia. Ciência e Tecnologia de Alimentos, Campinas, v. 27, p. 99-102, 2007. http://dx.doi.org/10.1590/S010120612007000500018.

PAPAZIAN, R. Sulfites: safe for most, dangerous for some. FDA Consumer Magazine, Rockville, v. 30, n. 10, p. 10-15, 1996.

POPOLIM, W. D. Análise de sulfitos em sucos de frutas e estimativa de seu consumo por escolares. 2009. 155 f. Tese (Doutorado em Nutrição Humana Aplicada)-Universidade de São Paulo, São Paulo, 2009.
RATHI, A.; RAJOR, H. J. K.; SHARMZA, R. K. Photodegradation of direct yellow-12 using $U V / \mathrm{H}_{2} \mathrm{O}_{2} / \mathrm{Fe}^{2+}$. Journal of Hazardous Materials, Amsterdam, v. 102, n. 2-3, p. 231-241, 2003. http:// dx.doi.org/10.1016/S0304-3894(03)00213-9. PMid:12972240.

SARTORI, J. A. S.; GALAVERNA, R.; EBERLIN, M. N.; CORREA, N. T.; MANDRO, J. L.; AGUIAR, C. L. Elucidation of color reduction involving precipitation of non-sugars in sugarcane (Saccharum sp.) juice by Fourier-Transform ion cyclotron resonance mass spectrometry. Journal of Food Processing and Preservation, Westport, v. 39, n. 6, p. 18261831, 2015.

TEÓFILO, R. F.; FERREIRA, M. M. C.; QUIMIOMETRIA, I. I. Planilhas eletrônicas para cálculos de planejamentos experimentais, um tutorial. Química Nova, São Paulo, v. 29, n. 2, p. 338-350, 2006. 